\title{
First international descriptive and interventional survey for cholesterol and non-cholesterol sterol determination by gas- and liquid- chromatography-Urgent need for harmonisation of analytical methods
}

\author{
Dieter Lütjohann $^{\mathrm{a}, *}$, Ingemar Björkhem ${ }^{\mathrm{b}, 1}$, Silvia Friedrichs ${ }^{\mathrm{a}, 1}$, Anja Kerksiek ${ }^{\mathrm{a}, 1}$,
} Anita Lövgren-Sandblom ${ }^{\mathrm{b}, 1}$, Wolf-Jochen Geilenkeuser ${ }^{\mathrm{c}, 1}$, Robert Ahrends ${ }^{\mathrm{d}}$, Isabel Andrade ${ }^{\mathrm{e}}$, Diana Ansorena $^{\mathrm{f}}$, Iciar Astiasarán ${ }^{\mathrm{f}}$, Lucía Baila-Rueda ${ }^{\mathrm{g}}$, Bianca Barriuso ${ }^{\mathrm{f}}$, Susen Becker ${ }^{\mathrm{h}, \mathrm{i}}$, Lionel Bretillon, Richard W. Browne ${ }^{\mathrm{k}}$, Claudio Caccia, ${ }^{\mathrm{j}, \mathrm{m}}$, Uta Ceglarek ${ }^{\mathrm{h}}$, Ana Cenarro ${ }^{\mathrm{g}}$, Peter J. Crick ${ }^{\mathrm{n}}$, Günter Fauler ${ }^{\mathrm{O}}$, Guadalupe Garcia-Llatas ${ }^{\mathrm{p}}$, Robert Gray ${ }^{\mathrm{q}}$, William J. Griffiths ${ }^{\mathrm{n}}$, Helena Gylling ${ }^{\mathrm{r}}$, Scott Harding ${ }^{\mathrm{q}, \mathrm{s}}$, Christin Helmschrodt ${ }^{\mathrm{h}, \mathrm{t}}$, Luigi Iuliano ${ }^{\mathrm{u}}$, Hans-Gerd Janssen ${ }^{\mathrm{v}}$, Peter Jones $^{\mathrm{w}}$, Leena Kaipiainen ${ }^{\mathrm{r}}$, Frank Kannenberg ${ }^{\mathrm{x}}$, María Jesús Lagarda ${ }^{\mathrm{p}}$, Valerio Leoni ${ }^{1, \mathrm{~m}}$, Ana Maria Lottenberg ${ }^{\mathrm{y}}$, Dylan S. MacKay ${ }^{\mathrm{w}}$, Silke Matysik ${ }^{\mathrm{z}}$, Jeff McDonald ${ }^{\mathrm{A}}$, Maria Menendez-Carreñov ${ }^{\text {, Semone B. Myrie }}{ }^{\mathrm{w}}$, Valéria Sutti Nunes ${ }^{\mathrm{y}}$, Richard E. Ostlund ${ }^{\mathrm{B}}$, Eliana Polisecki ${ }^{\mathrm{C}}$, Fernando Ramos ${ }^{\mathrm{D}}$, Todd C. Rideout ${ }^{\mathrm{E}}$, Ernst J. Schaefer ${ }^{\mathrm{C}}$, Gerd Schmitz ${ }^{\mathrm{Z}}$, Yuqin Wang $^{\mathrm{n}}$, Chiara Zerbinati ${ }^{\mathrm{u}}$, Ulf Diczfalusy ${ }^{\mathrm{b}, 1}$, Hans-Frieder Schött ${ }^{\mathrm{a}, \mathrm{d}, 1}$

${ }^{a}$ Institute of Clinical Chemistry and Clinical Pharmacology, University Hospital, Bonn, Germany

${ }^{\mathrm{b}}$ Department of Laboratory Medicine, Division of Clinical Chemistry, Karolinska University Hospital, Karolinska Institutet, Huddinge, Sweden

${ }^{\mathrm{c}}$ German Reference Institute for Bioanalytics, Bonn, Germany

${ }^{\mathrm{d}}$ Leibniz-Institut für Analytische Wissenschaften-ISAS-e.V., Dortmund, Germany

${ }^{\mathrm{e}}$ ESTESC-Coimbra Health School, Coimbra, Portugal

${ }^{\mathrm{f}}$ Department of Nutrition, Food Science and Physiology, Faculty of Pharmacy and Nutrition, University of Navarra, Pamplona, Spain

${ }^{\mathrm{g}}$ Hospital Universitario Miguel Servet, IIS Aragón, CIBERV, Zaragoza, Spain

${ }^{\mathrm{h}}$ Institute of Laboratory Medicine, Clinical Chemistry and Molecular Diagnostics, University Hospital Leipzig, Leipzig, Germany

${ }^{\mathrm{i}}$ Department of Pediatric Surgery, University of Leipzig, Leipzig, Germany

${ }^{\mathrm{j}}$ Centre des Sciences du Goŭt et de l'Alimentation, AgroSup Dijon, CNRS, INRA, Université Bourgogne Franche-Comté, Dijon, France

${ }^{\mathrm{k}}$ Biotechnical and Clinical Laboratory Sciences, Jacobs School of Medicine and Biomedical Sciences, University at Buffalo, Buffalo, NY, United States

${ }^{1}$ Laboratory of Clinical Chemistry, Hospital of Varese, ASST-Settelaghi, Varese, Italy

${ }^{\mathrm{m}}$ Laboratory of Clinical Pathology, Foundation IRCCS Istituto Neurologico Carlo Besta, Milan, Italy

${ }^{\mathrm{n}}$ Institute of Life Science, Swansea University Medical School, Swansea, United Kingdom

${ }^{\circ}$ Clinical Institute of Medical and Chemical Laboratory Diagnostics, Medical University of Graz, Graz, Austria

${ }^{\mathrm{P}}$ Nutrition and Food Science Area, University of Valencia, Burjassot, Valencia, Spain

${ }^{\mathrm{q}}$ Department of Nutritional Sciences, Faculty of Life Sciences \& Medicine King's College London, London, UK

${ }^{\mathrm{r}}$ University of Helsinki and Helsinki University Central Hospital, Internal Medicine and Abdominal Center, Helsinki, Finland

${ }^{s}$ Department of Biochemistry, Faculty of Science, Memorial University of Newfoundland, St. John's, Canada

${ }^{\mathrm{t}}$ Institute of Pharmacology, Pharmacy and Toxicology VMF, University of Leipzig, Leipzig, Germany

${ }^{u}$ Department of Medico-Surgical Sciences and Biotechnology, Vascular Biology and Mass Spectrometry Laboratory, Sapienza University of Rome, Latina, Italy

${ }^{\mathrm{v}}$ Unilever Research and Development, Analytical Sciences, Vlaardingen, the Netherlands

${ }^{\mathrm{w}}$ Richardson Centre for Functional Foods and Nutraceuticals, University of Manitoba, Winnipeg, Manitoba, Canada

${ }^{\mathrm{x}}$ Centrum für Laboratoriumsmedizin, Zentrallaboratorium, Universitätsklinikum Münster, Münster, Germany

${ }^{\mathrm{y}}$ Faculty of Medical Sciences, Endocrinology and Metabolism Division, University of Sao Paulo, Sao Paulo, Brazil

${ }^{\mathrm{z}}$ Institute of Clinical Chemistry and Laboratory Medicine, University Hospital Regensburg, Regensburg, Germany

\footnotetext{
Abbreviations: $5 \alpha$-chol, $5 \alpha$-cholestane; epi, epicoprostanol 5 $\alpha$-cholestan-3 $\alpha$-ol; FID, flame ionization detection; GLC, gas-liquid chromatography; LC, liquid chromatography; MSD, mass selective detector; NCS, non-cholesterol sterols; RfB, German Reference Institute for Bioanalytics

*Corresponding author at: Institute for Clinical Chemistry and Clinical Pharmacology, University Clinics Bonn, Sigmund-Freud-Strasse 25, D-53127, Bonn, Germany.

E-mail address: dieter.luetjohann@ukbonn.de (D. Lütjohann).
} 


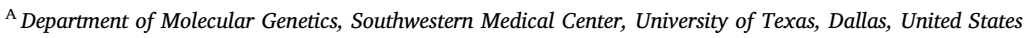

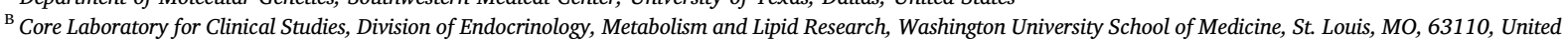

States

${ }^{\mathrm{C}}$ Boston Heart Diagnostics, Framingham, MA, United States

${ }^{D}$ REQUIMTE/LAQV, Health Sciences Campus, Faculty of Pharmacy, University of Coimbra, Coimbra, Portugal

${ }^{\mathrm{E}}$ Department of Exercise and Nutrition Sciences, School of Public Health and Health Professions, University of Buffalo, Bufalo, NY, United States

\section{A R T I C L E I N F O}

\section{Keywords:}

Surrogate marker

Cholesterol absorption

Cholesterol synthesis

Cholesterol balance

Atherosclerosis

Phytosterols

\begin{abstract}
A B S T R A C T
Serum concentrations of lathosterol, the plant sterols campesterol and sitosterol and the cholesterol metabolite $5 \alpha$-cholestanol are widely used as surrogate markers of cholesterol synthesis and absorption, respectively. Increasing numbers of laboratories utilize a broad spectrum of well-established and recently developed methods for the determination of cholesterol and non-cholesterol sterols (NCS). In order to evaluate the quality of these measurements and to identify possible sources of analytical errors our group initiated the first international survey for cholesterol and NCS. The cholesterol and NCS survey was structured as a two-part survey which took place in the years 2013 and 2014. The first survey part was designed as descriptive, providing information about the variation of reported results from different laboratories. A set of two lyophilized pooled sera (A and B) was sent to twenty laboratories specialized in chromatographic lipid analysis. The different sterols were quantified either by gas chromatography-flame ionization detection, gas chromatography- or liquid chromatography-mass selective detection. The participants were requested to determine cholesterol and NCS concentrations in the provided samples as part of their normal laboratory routine. The second part was designed as interventional survey. Twenty-two laboratories agreed to participate and received again two different lyophilized pooled sera (C and D). In contrast to the first international survey, each participant received standard stock solutions with defined concentrations of cholesterol and NCS. The participants were requested to use diluted calibration solutions from the provided standard stock solutions for quantification of cholesterol and NCS. In both surveys, each laboratory used its own internal standard ( $5 \alpha$-cholestane, epicoprostanol or deuterium labelled sterols).

Main outcome of the survey was, that unacceptably high interlaboratory variations for cholesterol and NCS concentrations are reported, even when the individual laboratories used the same calibration material. We discuss different sources of errors and recommend all laboratories analysing cholesterol and NCS to participate in regular quality control programs.
\end{abstract}

\section{Introduction}

Serum or plasma concentrations of the cholesterol precursors lanosterol, lathosterol, and desmosterol are widely used as surrogate markers of endogenous cholesterol synthesis [1]. The cholesterol metabolite $5 \alpha$-cholestanol and the plant sterols campesterol and sitosterol are used as markers of cholesterol absorption [2,3]. These non-cholesterol sterols (NCS) show even stronger correlations with cholesterol absorption and synthesis when expressed as ratios to total cholesterol, which standardizes for variations in sterol transport protein concentrations [4]. Specifically, when reporting NCS as their ratios to cholesterol, the cholesterol measurement should ideally be performed from the same sample preparation as for the NCS analysis. The interest in surrogate markers of cholesterol metabolism is recently increasing and more laboratories develop new methods for the determination of cholesterol and NCS using different chromatographic separation and mass spectrometric detection methods. Conflicting absolute and cholesterol-corrected NCS concentrations are reported in the literature making it difficult or nearly impossible to define cut-off values [5-7] or to compare absolute or cholesterol corrected values from different studies in meta-analysis. For a better comparison of reported values and the identification of methodological sources of errors, we planned and performed the first international surveys for cholesterol and NCS. The first part of the cholesterol and NCS survey was initiated in the year 2013 under the expertise of the German Reference Institute for Bioanalytics (RfB) and the Laboratory for Specialized Lipid Diagnostic of the University Hospital, both located in Bonn, Germany. The aim of the first survey was to investigate the variations in cholesterol and NCS

\footnotetext{
${ }^{1}$ These authors contributed in setting up the protocol (IB, DL, UD, H-FS), preparing standard solutions (UD, AL-S, AK, SF), preparing and distributing the lyophilized standard samples A-D (W-JG, H-FS, DL), evaluating the data (W-JG, H-FS, DL), calculating statistics (W-JG, H-FS, DL), and finally in preparing the first draft of the manuscript (IB, DL, UD, H-FS).
}

concentrations routinely determined by different laboratories in Europe, North and South America. Here, we reflect the comparability of cholesterol and NCS concentrations determined by different separation and detection methods and discuss the suitability of different compounds used as internal standards for sterol quantification. Twenty laboratories specialized in chromatographic lipid analysis, by either gasor liquid-chromatography were enrolled to participate in this first survey part. The second part of the international cholesterol and neutral sterol survey took place one year later in 2014. Twenty-two laboratories participated in the second survey whereof five laboratories attended for the first time. Contrary to the first survey, the second was designed as an interventional trial. The focus was on the influence of the utilized calibration solutions and the participants were requested to use provided stock solutions for the quantification of the sample material.

\section{Materials and methods}

The participants submitted results from six different methods for sterol determination: capillary gas chromatography-flame ionisation detection (GC-FID) with either $5 \alpha$-cholestane ( $5 \alpha$-chol) or epicoprostanol (epi) as internal standard (GC-FID-5 $\alpha$-chol and GC-FID-epi, respectively), capillary GC-mass selective detection (MSD) with either $5 \alpha$ chol, epi or deuterium labelled sterols as internal standards (GC-MSD5 $\alpha$-chol, GC-MSD-epi and GC-MSD-deuterium, respectively) and high performance-liquid chromatography (HPLC) with MSD and deuterated sterols (LC-MSD-deuterium) used as internal standard. Since each laboratory was requested to use its specific routine analytical method, the work-up procedure and determination setting are strikingly different for the individual participants. Table 1 shows an overview of the reported sample work-up conditions, chemicals, chromatographic columns, and instrumentation for GC analyses used in the surveys. Most of the laboratories used alkaline hydrolysis in order to deconjugate fatty acid esterified sterols and thus analysing total serum sterol concentrations after derivatisation of the free hydroxyl groups. One laboratory, 
Table 1

Methodological parameters and instrumental equipment from the different contributors.

\begin{tabular}{|c|c|}
\hline sample volume $[\mu \mathrm{L}]$ & $10 ; 20 ; 40 ; 50 ; 100 ; 200 ; 250 ; 300 ; 400 ; 500 ; 1000$ \\
\hline hydrolysis time [min] & $15 ; 20 ; 60 ; 65 ; 68 ; 70 ; 90 ; 120$ \\
\hline hydrolysis temperature $\left[{ }^{\circ} \mathrm{C}\right]$ & $50 ; 60 ; 80 ; 90$ \\
\hline hydrolysis $\mathrm{NaOH} / \mathrm{KOH}$ concentration $[\mathrm{mol} / \mathrm{L}]$ & $0.35 ; 0.5 ; 0.71 ; 1.0 ; 1.78 ; 2.0$ \\
\hline extraction solvents & hexane; petroleum ether; petroleum ethyl ether; chloroforme; tetrachlorethylene; cyclohexane; isooctane; heptane \\
\hline solvent volume for extraction $[\mathrm{mL}]$ & $2 ; 3 ; 4 ; 5 ; 9 ; 20$ \\
\hline silylation time [min] & 30; 45; 60; over night \\
\hline silylation temperature $\left[{ }^{\circ} \mathrm{C}\right]$ & $37 ; 55 ; 60 ; 70$ \\
\hline silylation reagents (GC only) & $\begin{array}{l}\text { HMDS/TMCS/pyridine (3:1:9)(Supelco) dimethylformamide/HMDS/TMCS Sylon BFT BSTFA/TMCS 99:1 + pyridine TriSil reag } \\
\text { (Thermo Fischer) HMDS/TMCS/Pyridine 2:1:3 MSTFA }\end{array}$ \\
\hline Injection volume $[\mu \mathrm{L}]$ & $1 ; 2 ; 3 ; 25 ; 40$ \\
\hline split mode injection (GC only) & $1: 3 ; 10: 1 ; 12: 1 ; 15: 1 ; 20: 1$; splitless \\
\hline column lenght (GC only) $[\mathrm{m}]$ & $25 ; 30$ \\
\hline column diameter (GC only) $[\mathrm{mm}]$ & $0.22 ; 0.25 ; 0.32$ \\
\hline column film thickness (GC only) $[\mu \mathrm{m}]$ & $0.1 ; 0.25$ \\
\hline columns (GC only) & $\begin{array}{l}\text { SUPELCO SAC }{ }^{\mathrm{TM}}-5 \text { Capillary GC Column HP-Ultra2 SAC-5 capilary column DB-5-MS Restek RXI } 1 \mathrm{mS} \text { (100\% dimethyl } \\
\text { polysiloxane) CP_SIL_5CB HAT-5 carbone modified siloxane HP-5MS 5\% phenylmethyl siloxane }\end{array}$ \\
\hline separation instruments (GC only) & GC-Agilent Technologies 6890 N GC-Agilent 7890A GC-Shimazu GC-17A FID GC-HP4890 Shimadzu GC-MSQP2010 \\
\hline detectors & $\begin{array}{l}\text { Flame ionisation detector Mass selective detector 5975c inert xl ms Ion Trap AgilentMS } 5973 \text { N } 4000 \text { Qtrap triple quad SCIEX } \\
\text { API } 3000 \text { triple quadrupol Photospray Thermo TSQ8000 Triple quad }\end{array}$ \\
\hline
\end{tabular}

using LC-MSD, quantified free and esterified fraction of the freeze-dried test-samples in order to calculate the total concentration. Some laboratories used fully automated peak integration and quantitation software belonging to the software package of the supplier. Others integrated the individual peaks half-automatically using self-created integration and evaluation macros with the option to supervise and correct integration of each peak. It should be emphasized that the individual sample work-up procedures or detection specifications cannot be reported within this article because of the anonymization of the participants. Since the freeze-dried serum samples were routinely used in round-robin tests of the RfB, it was possible to give a reference value (target concentration) only for cholesterol. The lyophilized serum samples were additionally used to measure other parameters in clinical routine biochemistry such as triglycerides, total protein or subspecies proteins such as albumin or gamma globuline in additional ring trials in Germany. All these parameters were within the normal range as characterized by reference values except for triglycerides in sample D of the second ring trial, where the median level was marginally increased (data not shown). Single values more than $50 \%$ different from the mean value of all submitted sterol concentrations have a marked effect on the mean values of the whole participant group. Therefore, individual values more than $50 \%$ different from the mean value were regarded as outliers and several of the above-mentioned parameters were additionally calculated without the outlier values and reported within the script. The guidelines of the German Medical Association for quality assurance of clinical laboratory investigations tolerates relative deviations for cholesterol within external proficiency testing of up to $13 \%$ [8]. Due to the relatively small number of participants in our surveys we set a relative deviation, that can be considered as acceptable to $\pm 15 \%$ of the mean value of the included participants. The mean values, standard deviation, minimum and maximum values of all participants as well as the ratios of $5 \alpha$-cholestanol, lathosterol, campesterol to cholesterol and the ratio lathosterol to campesterol are listed in Tables 2 and 3 and shown in Figs. 1 and 2.

\subsection{The first international cholesterol and NCS survey}

Twenty laboratories specialized in chromatographic lipid analysis participated in this survey. A set of two different lyophilized pooled sera (sample A and B) was sent to each participant and analysed with the individual determination method during the routine operation of each laboratory. After a period of twelve weeks the participants were requested to submit their results to RfB for further data analysis. Eighteen of the twenty participating laboratories submitted their results before the 12-week deadline and were included for further evaluation.
The data were submitted in the units individually used by the participant $(\mathrm{ng} / \mathrm{mL}, \mathrm{mg} / \mathrm{mL}, \mu \mathrm{g} / \mathrm{dL}, \mathrm{mg} / \mathrm{dL}, \mathrm{mg} / \mathrm{L}, \mu \mathrm{mol} / \mathrm{L}, \mathrm{mmol} / \mathrm{L})$ and converted into standard units for comparison (cholesterol and NCS as $\mathrm{mg} / \mathrm{dL}$ ). Each participant received the results presented in form of Youden-Plots and basic statistical data. The evaluated data were given in the individual units, which were used by the participant.

\subsection{The second international cholesterol and NCS survey}

Twenty-two laboratories participated in the second survey whereof the results of five laboratories were included that attended for the first time. Two groups that attended the first survey were not able to participate in the second survey. Again a set of two different lyophilized pooled sera (sample $C$ and $D$ ) was sent to each participant and analysed with the individual determination method used in the routine operation of each laboratory. Contrary to the first survey the second part was designed as an interventional trial with focus on the influence of the utilized calibration solutions on cholesterol and NCS concentrations. Therefore, the participants were requested to use the in glass ampoules provided stock solutions (containing cholesterol, $5 \alpha$-cholestanol, lathosterol, campesterol, and sitosterol in the concentrations $1.0 \mathrm{mg} / \mathrm{mL}$; $18.8 \mu \mathrm{g} / \mathrm{mL}, 18.8 \mu \mathrm{g} / \mathrm{mL}, 13.0 \mu \mathrm{g} / \mathrm{mL}$, and $18.0 \mu \mathrm{g} / \mathrm{mL}$; respectively). The stock solutions for the quantification of the sample material were generously provided by the Department of Laboratory Medicine, Division of Clinical Chemistry, Karolinska University Hospital, Karolinska Institutet, Huddinge, Sweden. All non-cholesterol sterols for the quantification of the sample material were purchased from SigmaAldrich. Data collection and result transmission procedure was identically to the above described protocol of the first survey part.

\subsection{Statistical analysis}

Statistical parameters (mean values, standard deviations, coefficients of variation, maximum and minimum values) of the submitted concentrations of all participating laboratories were calculated. Conditions that impede a clear and strong statistical evaluation of the data set are the fact that different laboratories participated in the two surveys (only twelve laboratories participated in both surveys). For that reason, only a qualitative discussion of the data set is performed.

\section{Results and discussion}

The results and subgroup analysis of cholesterol and NCS from the both surveys are listed in Table 2 and shown in Fig. 1, A1 to E2. 


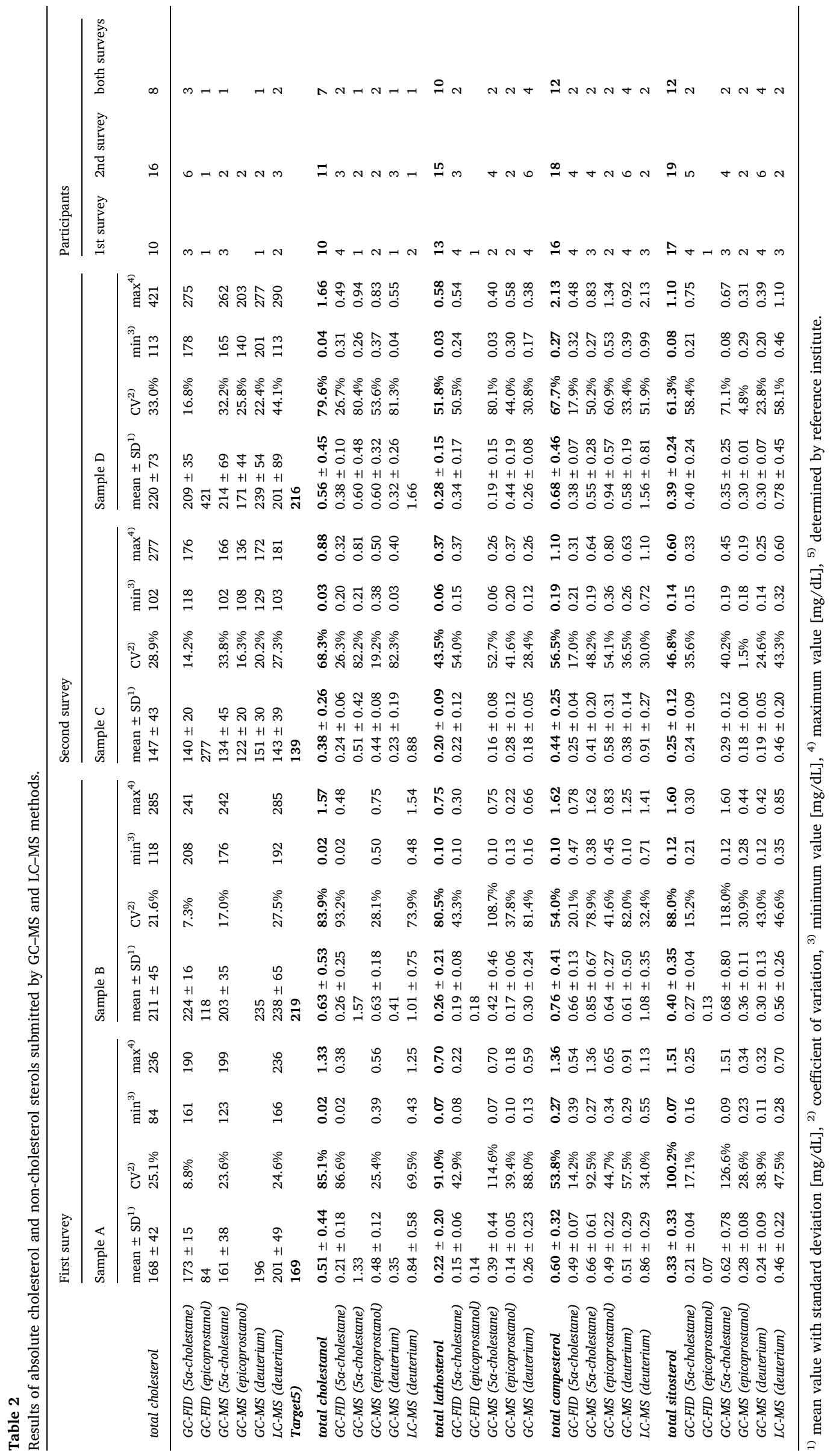




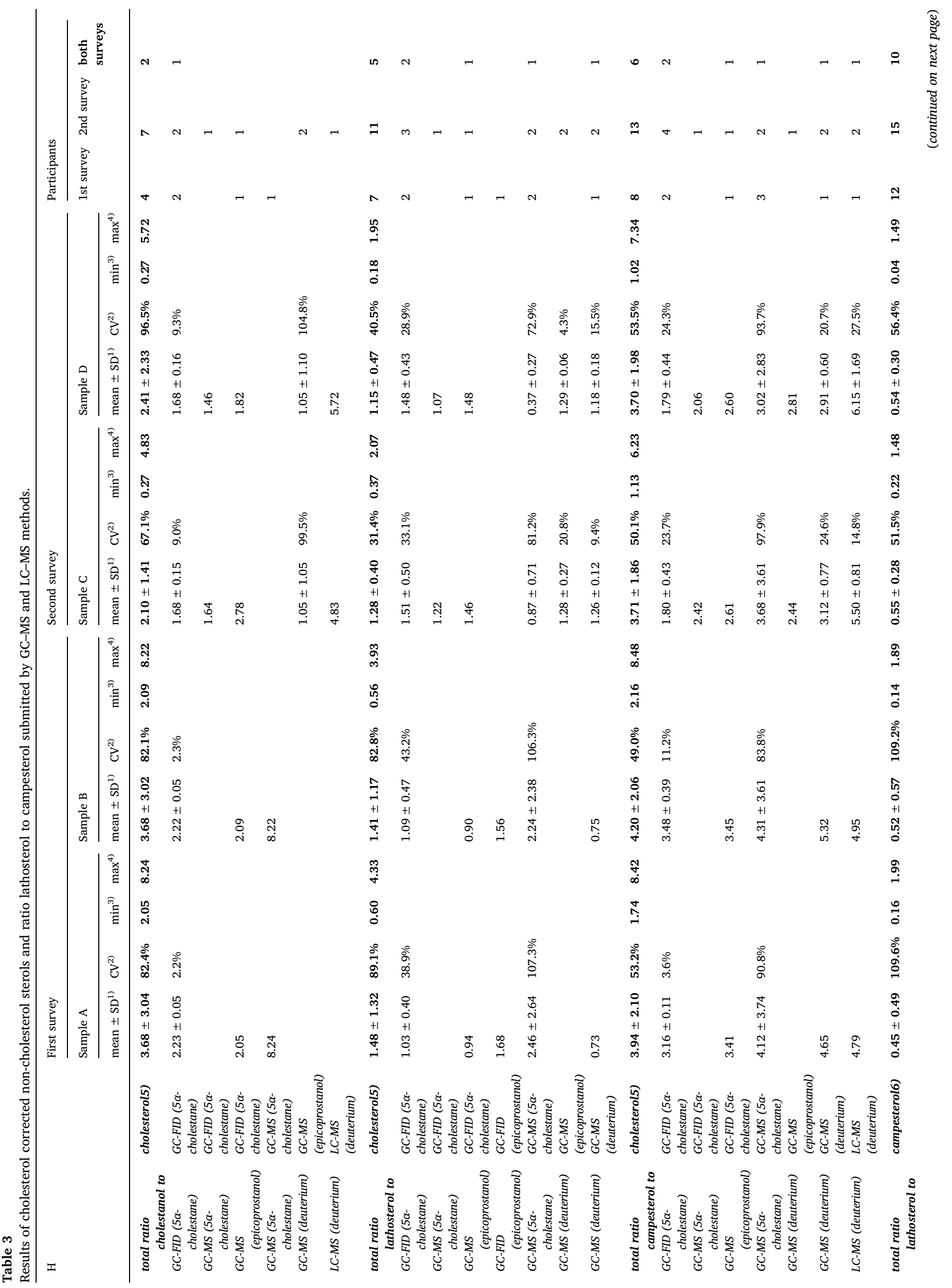




\subsection{Cholesterol}

In the first survey for chromatographically determined cholesterol ten different laboratories participated. The mean cholesterol concentrations determined by all ten participants were calculated for sample A (168 $\pm 42 \mathrm{mg} / \mathrm{dL} ; \mathrm{CV} 25.1 \%)$ and B (211 $\pm 45 \mathrm{mg} / \mathrm{dL} ; \mathrm{CV}$ $21.6 \%$ ). The target concentrations specified by the RfB were $169 \mathrm{mg} / \mathrm{dL}$ (sample A) and $219 \mathrm{mg} / \mathrm{dL}$ (sample B). Five out of ten participants did not determine cholesterol concentrations within a $\pm 15 \%$ range of the target value. One participant was identified as outlier (Fig. 1A1, a).

In the second survey part sixteen different laboratories attended from which eight participated in both surveys. The mean cholesterol concentrations determined by all sixteen participants were calculated for sample C (147 $\pm 43 \mathrm{mg} / \mathrm{dL}$; CV 28.9\%) and D $(220 \pm 73 \mathrm{mg} / \mathrm{dL}$; CV $33.0 \%)$. The target concentrations specified by the reference institute were $139 \mathrm{mg} / \mathrm{dL}$ (sample C) and $216 \mathrm{mg} / \mathrm{dL}$ (sample D). Only six out of sixteen participants reported cholesterol values that are within a $\pm 15 \%$ range of our accepted target value. One participant was identified as outlier (Fig. 1A2, a).

The differences in reported cholesterol results are difficult for an interpretation. Probably insufficient fatty acid deconjugation of the cholesterol esters during alkaline hydrolysis might have impaired the analysis of some participants. It is noteworthy that even with methods based on isotope dilution GC-MSD and use of an deuterated internal standard and the same calibration material two laboratories reported a difference of about $25 \%$. Since our survey shows that two of the three laboratories using GC-FID reported excellent results, the present data does not enable the conclusion that deuterium labelled cholesterol used as internal standard is superior to the use of $5 \alpha$-cholestane or epicoprostanol. The greatest variations in cholesterol determination were obtained using methods based on LC-MSD.

\section{2. $5 \alpha$-cholestanol}

In the first survey for chromatographic analysis of serum $5 \alpha$-cholestanol ten different laboratories participated. The mean $5 \alpha$-cholestanol concentration determined by all ten participants were calculated for sample A $(0.515 \pm 0.438 \mathrm{mg} / \mathrm{dL} ; \quad \mathrm{CV} \quad 85.1 \%)$ and B $(0.632 \pm 0.530 \mathrm{mg} / \mathrm{dL} ; \mathrm{CV} 83.9 \%)$. Therefore, none of the participants determined $5 \alpha$-cholestanol concentrations within a $\pm 15 \%$ range of the mean value calculated of the reported of all participants. The concentrations submitted by four out of ten laboratories were identified as outliers (Fig. 1 B1, a to d) and new corrected mean concentrations for the remaining six laboratories were calculated (sample A: $0.410 \pm 0.079 \mathrm{mg} / \mathrm{dL}, \mathrm{CV} 19.2 \%$; sample B: $0.517 \pm 0.120 \mathrm{mg} / \mathrm{dL}$, CV 23.1\%). Out of these remaining six laboratories, four determined $5 \alpha$-cholestanol concentrations within a $\pm 15 \%$ range of the corrected mean value.

In the second survey eleven different laboratories attended from which seven laboratories participated in both surveys. The mean $5 \alpha-$ cholestanol concentrations determined by eleven participants were calculated for sample C $(0.380 \pm 0.260 \mathrm{mg} / \mathrm{dL} ; \mathrm{CV} 68.3 \%)$ and D $(0.559 \pm 0.445 \mathrm{mg} / \mathrm{dL} ; \mathrm{CV} 79.6 \%)$. One of the participants determined $5 \alpha$-cholestanol concentrations within a $\pm 15 \%$ range of the mean value of all participants and four participants reported results which are identified as outliers (Fig. 1 B2, a to d). The new corrected mean concentration for the remaining seven laboratories was calculated as (sample C: $\quad 0.322 \pm 0.111 \mathrm{mg} / \mathrm{dL}, \quad$ CV $\quad 34.4 \%$; sample D: $0.465 \pm 0.183 \mathrm{mg} / \mathrm{dL}, \mathrm{CV} 39.4 \%$ ). Only one out of the seven remaining laboratories reported results within a $\pm 15 \%$ range of the corrected mean value.

The first survey revealed a 20 -fold variation in the individual results between the highest and lowest reported concentration. Also, the second survey revealed widely scattered concentrations with four distinct outliers. Even after the exclusion of these outliers, a 2.5 fold variation between the highest and lowest included concentrations 

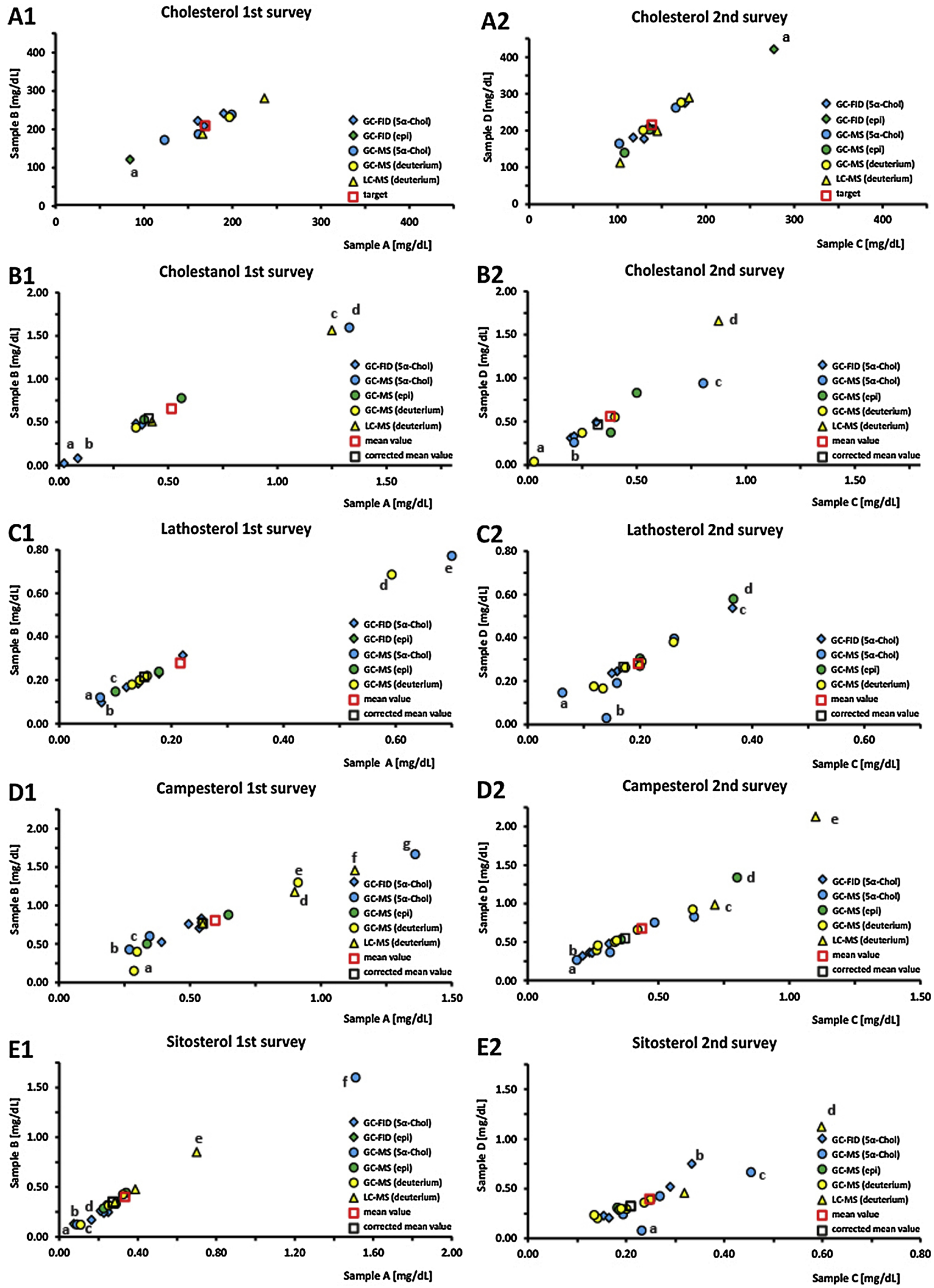

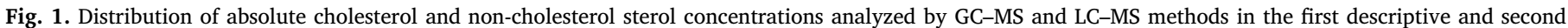
interventional survey. Values a-e in the figures A1-E2 are regarded as outliers. 

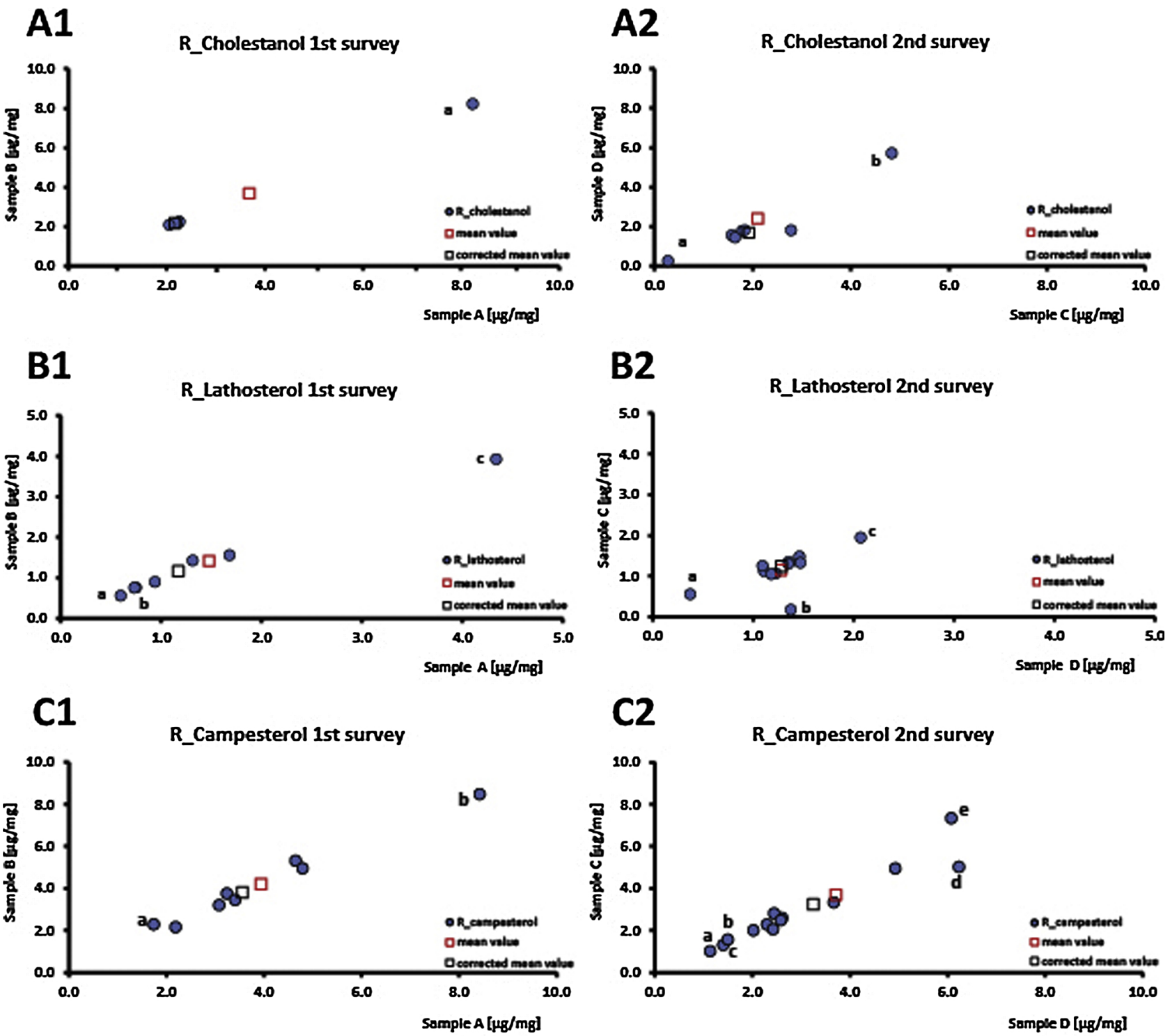

\section{D1}

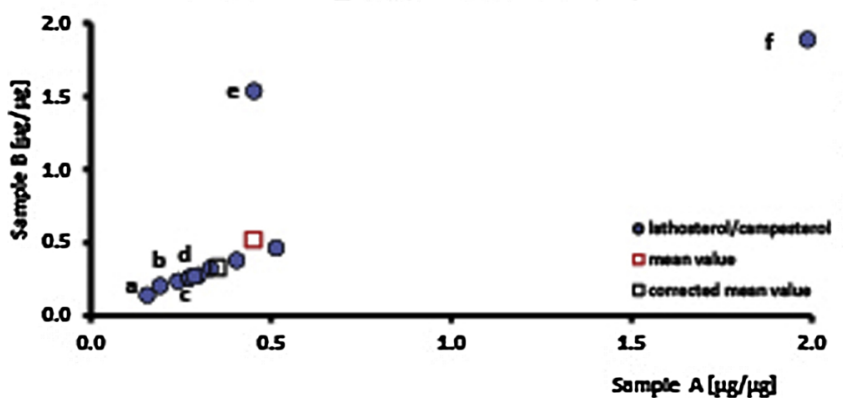

\section{D2 Lathosterol_Campesterol 2nd survey}

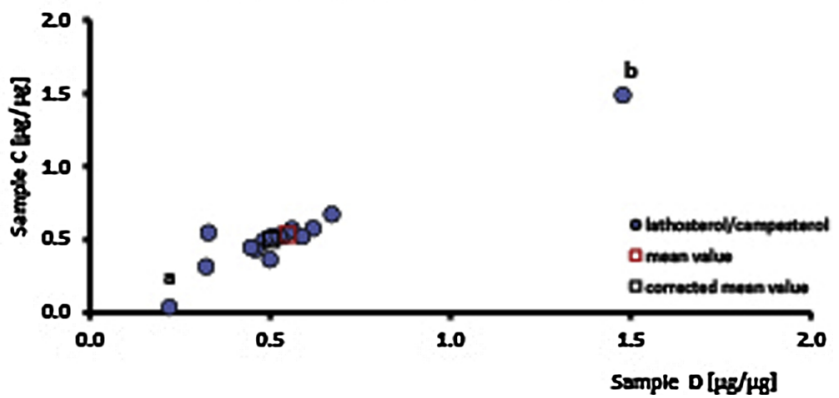

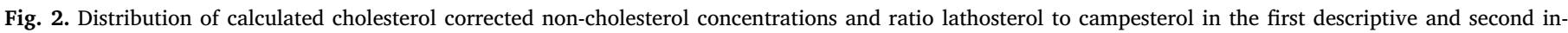
terventional survey. Values a-e in the figures A1-D2 are regarded as outliers.

persisted in the second survey. One of the major problems with the $5 \alpha$ cholestanol determination may be related to the fact that $5 \alpha$-cholestanol has very similar chromatographic properties to cholesterol, whose concentration in serum are 300 fold higher. Therefore, the separation performance of the chromatographic system may be a critical for a reliable $5 \alpha$-cholestanol quantification.

\subsection{Lathosterol}

In the first part of the survey for assay of lathosterol thirteen different laboratories participated. The mean lathosterol concentrations determined by all thirteen participants were calculated for sample A $(0.216 \pm 0.197 \mathrm{mg} / \mathrm{dL} ; \mathrm{CV} 91.0 \%)$ and B $(0.257 \pm 0.207 \mathrm{mg} / \mathrm{dL} ; \mathrm{CV}$ $80.5 \%)$. None of the participants reported lathosterol levels within 
a $\pm 15 \%$ range of the mean value of all participants. The concentrations submitted by five of the thirteen laboratories were regarded as outliers (Fig. $1 \mathrm{C} 1$, a and e) and new, corrected mean concentrations for the remaining eleven laboratories were calculated (sample A: $0.158 \pm 0.033 \mathrm{mg} / \mathrm{dL}, \mathrm{CV} 20.6 \%$; sample B: $0.201 \pm 0.044 \mathrm{mg} / \mathrm{dL}$, CV 21.7\%). After the correction for the outlier values five of the remaining eleven laboratories reported lathosterol concentrations within a $\pm 15 \%$ range of the corrected mean value.

In the second survey fifteen different laboratories attended from which ten laboratories participated in both surveys. The mean lathosterol concentration obtained from all fifteen participants were calculated for sample C $(0.197 \pm 0.086 \mathrm{mg} / \mathrm{dL} ; \mathrm{CV} 43.5 \%)$ and D $(0.281 \pm 0.145 \mathrm{mg} / \mathrm{dL} ; \mathrm{CV} 51.8 \%)$. Four of the participants determined lathosterol concentrations within a $\pm 15 \%$ range of the mean value of all participants. In the second survey, four participants were identified as outliers (Fig. 1 C2, a to d) and new corrected mean concentrations for the remaining eleven laboratories were calculated (sample C: $\quad 0.183 \pm 0.047 \mathrm{mg} / \mathrm{dL}, \quad$ CV $\quad 25.5 \%$; sample D: $0.265 \pm 0.076 \mathrm{mg} / \mathrm{dL}, \mathrm{CV} 28.5 \%$ ). Only five of the remaining laboratories reported results within $\mathrm{a} \pm 15 \%$ range of the common mean value.

In both surveys very high variations of lathosterol concentrations were reported. After exclusion of 4 outliers in the second survey a 2.0 fold variation between the reported results remained.

While chromatographic separation of $5 \alpha$-cholestanol in presence of high serum cholesterol concentrations is very challanging, lathosterol can be chromatographically well separated from other non-cholesterol sterols. However, incorrect peak identification and/or incorrect integration of the peak could lead to these discrepancies in the results reported from the different laboratories.

\subsection{Campesterol}

In the first survey for chromatographically determined campesterol sixteen different laboratories participated. The mean campesterol concentrations determined by all sixteen participants were calculated for sample A $(0.597 \pm 0.321 \mathrm{mg} / \mathrm{dL} ; \quad$ CV $53.8 \%)$ and B $(0.760 \pm 0.410 \mathrm{mg} / \mathrm{dL} ; \mathrm{CV} 54.0 \%)$. Five of the participants reported campesterol concentrations within a $\pm 15 \%$ range of the mean value of all participants. The concentrations reported by seven of the sixteen laboratories were identified as outliers (Fig. 1 D1, a to g) and new corrected mean concentrations for the remaining eleven laboratories were calculated (sample A: $0.488 \pm 0.106 \mathrm{mg} / \mathrm{dL}, \mathrm{CV} 21.8 \%$; sample B: $0.656 \pm 0.134 \mathrm{mg} / \mathrm{dL}, \mathrm{CV} 20.4 \%$ ). Four of these remaining nine laboratories determined campesterol concentrations within a $\pm 15 \%$ range of the corrected mean value.

In the second survey, eighteen different laboratories participated from which twelve laboratories participated in both surveys. The mean campesterol concentrations determined by all eighteen participants were calculated as for sample C $(0.437 \pm 0.247 \mathrm{mg} / \mathrm{dL} ; \mathrm{CV} 56.5 \%)$ and $\mathrm{D}(0.677 \pm 0.458 \mathrm{mg} / \mathrm{dL} ; \mathrm{CV} 67.7 \%)$. Two of the eighteen participants determined campesterol concentrations within a $\pm 15 \%$ range of the mean value of all participants. In the second survey five participants were regarded as outliers (Fig. 1 D2, a to e) and corrected mean concentrations for the remaining thirteen laboratories were calculated (sample C: $\quad 0.373 \pm 0.135 \mathrm{mg} / \mathrm{dL}, \quad \mathrm{CV} \quad 36.1 \%$; sample $\quad \mathrm{D}$ : $0.549 \pm 0.187 \mathrm{mg} / \mathrm{dL}, \mathrm{CV} 33.9 \%)$. Only three of the remaining laboratories determined results within an $\pm 15 \%$ range of the corrected mean value.

For the measurement of campesterol very high variations were reported in both surveys. After exclusion of 5 outliers in the second survey there was still a 2.5 fold difference between the highest and lowest reported value.

\subsection{Sitosterol}

In the first survey part for chromatographically determined sitosterol seventeen different laboratories participated. The mean sitosterol concentrations determined by all seventeen participants were calculated for sample A $(0.334 \pm 0.335 \mathrm{mg} / \mathrm{dL} ; \mathrm{CV} 100.2 \%)$ and B $(0.401 \pm 0.353 \mathrm{mg} / \mathrm{dL} ; \mathrm{CV} 88.0 \%)$. Two of the participants determined sitosterol concentrations within a $\pm 15 \%$ range of the mean value of all participants. The concentrations submitted by six of the seventeen laboratories were identified as outliers (Fig. 1 E1, a to f) and new corrected mean concentrations for the remaining eleven laboratories were calculated (sample A: $0.276 \pm 0.055 \mathrm{mg} / \mathrm{dL}, \mathrm{CV} 20.1 \%$; sample B: $0.345 \pm 0.071 \mathrm{mg} / \mathrm{dL}, \mathrm{CV} 20.5 \%$ ). Of the remaining eleven laboratories four determined sitosterol concentrations within $\mathrm{a} \pm 15 \%$ range of the corrected mean value.

In the second survey, nineteen different laboratories participated from which twelve laboratories participated in both surveys. The mean sitosterol concentrations determined by all eighteen participants were calculated for sample C $(0.248 \pm 0.116 \mathrm{mg} / \mathrm{dL} ; \mathrm{CV} 46.8 \%)$ and D $(0.387 \pm 0.237 \mathrm{mg} / \mathrm{dL} ; \mathrm{CV} 61.3 \%)$. Only three of the nineteen participants reported sitosterol concentrations within a $\pm 15 \%$ range of the mean value of all participants. In the second survey four participants were identified as outliers (Fig. $1 \mathrm{E} 2$, a to d) and a corrected mean concentrations for the remaining sixteen laboratories were calculated (sample C: $\quad 0.206 \pm 0.055 \mathrm{mg} / \mathrm{dL}, \quad$ CV $26.6 \%$; sample D: $0.317 \pm 0.095 \mathrm{mg} / \mathrm{dL}, \mathrm{CV} 30.1 \%$ ). Only six of the remaining laboratories determined results within a $\pm 15 \%$ range of the corrected mean value.

Also, in both surveys a high variation in the reported sitosterol concentrations was observed. After removing 4 outliers in the second survey there was still a 2.0 fold difference between the highest and the lowest reported value.

\subsection{Ratios of 5 $\alpha$-cholestanol, lathosterol, and campesterol to cholesterol and lathosterol to campesterol}

Since the ratios of $5 \alpha$-cholestanol, lathosterol, and campesterol to cholesterol and lathosterol to campesterol are the most common surrogate markers for absorption and synthesis rates, we calculated these ratios for sample A to D for all participants. The results and subgroup analysis from the both surveys are listed in details in Table 2 and shown in Fig. $2 \mathrm{~A} 1$ to $\mathrm{D} 2$.

\subsection{1. $5 \alpha$-cholestanol to cholesterol}

In the first survey part four participants were included with calculated results for sample A $(3.68 \pm 3.04 \mu \mathrm{g} / \mathrm{mg}$; CV $82.4 \%)$ and B $(3.68 \pm 3.02 \mu \mathrm{g} / \mathrm{mg}$; CV $82.1 \%)$. None of the participants determined $5 \alpha$-cholestanol to cholesterol ratio within a $\pm 15 \%$ range of the mean value of all participants and one participant was identified as outlier (Fig. $2 \mathrm{~A} 1$, a). The new corrected mean value for the remaining three laboratories were calculated for A $(2.17 \pm 0.11 \mu \mathrm{g} / \mathrm{mg}$; CV 4.9\%) and B $(2.17 \pm 0.08 \mu \mathrm{g} / \mathrm{mg}$; CV 3.8\%). All the remaining laboratories determined the $5 \alpha$-cholestanol to cholesterol ratio within a $\pm 15 \%$ range of the corrected mean value.

In the second survey part seven participants were included with calculated results for sample A $(2.10 \pm 1.41 \mu \mathrm{g} / \mathrm{mg}$; CV 67.1\%) and B $(2.41 \pm 2.33 \mu \mathrm{g} / \mathrm{mg}$; CV $96.5 \%)$. None of the participants determined $5 \alpha$-cholestanol to cholesterol ratio within a $\pm 15 \%$ range of the mean value of all participants and two participants were identified as outliers (Fig. 2 A2, a and b). The new corrected mean value for the remaining five laboratories were calculated for A (1.92 $\pm 0.49 \mu \mathrm{g} / \mathrm{mg}$; CV 25.6\%) and B $(1.69 \pm 0.17 \mu \mathrm{g} / \mathrm{mg}$; CV $10.1 \%)$. Three of the remaining five laboratories determined the $5 \alpha$-cholestanol to cholesterol ratio within a $\pm 15 \%$ range of the corrected mean value. 


\subsubsection{Lathosterol to cholesterol}

In the first survey part seven participants were included with calculated results for sample A $(1.48 \pm 1.32 \mu \mathrm{g} / \mathrm{mg}$; CV $89.1 \%)$ and B $(1.41 \pm 1.17 \mu \mathrm{g} / \mathrm{mg}$; CV $82.8 \%)$. Two of the participants determined lathosterol to cholesterol ratio within a $\pm 15 \%$ range of the mean value of all participants and three participants were identified as outliers (Fig. $2 \mathrm{~B} 1$, a to c). The new corrected mean value for the remaining four laboratories were calculated for A (1.17 $\pm 0.41 \mu \mathrm{g} / \mathrm{mg}$; CV 35.4\%) and B $(1.16 \pm 0.39 \mu \mathrm{g} / \mathrm{mg} ;$ CV $33.7 \%)$. None of the laboratories determined the lathosterol to cholesterol ratio within a $\pm 15 \%$ range of the corrected mean value.

In the second survey part eleven participants were included with calculated results for sample A $(1.28 \pm 0.40 \mu \mathrm{g} / \mathrm{mg}$; CV $31.4 \%)$ and B $(1.15 \pm 0.47 \mu \mathrm{g} / \mathrm{mg}$; CV $40.5 \%)$. Five of the participants determined lathosterol to cholesterol ratio within a $\pm 15 \%$ range of the mean value of all participants and three participants were identified as outliers (Fig. 2 B2, a to c). The new corrected mean value for the remaining five laboratories were calculated for A $(1.28 \pm 0.15 \mu \mathrm{g} / \mathrm{mg} ; \mathrm{CV} 11.7 \%)$ and B $(1.25 \pm 0.15 \mu \mathrm{g} / \mathrm{mg}$; CV $12.2 \%)$. Six of the remaining eight laboratories determined the lathosterol to cholesterol ratio within a $\pm 15 \%$ range of the corrected mean value.

\subsubsection{Campesterol to cholesterol}

In the first survey part eight participants were included with calculated results for sample A $(3.94 \pm 2.10 \mu \mathrm{g} / \mathrm{mg}$; CV $53.2 \%)$ and B $(4.20 \pm 2.06 \mu \mathrm{g} / \mathrm{mg}$; CV $49.0 \%)$. None of the participants determined campesterol to cholesterol ratio within a $\pm 15 \%$ range of the mean value of all participants and two participants were identified as outliers (Fig. $2 \mathrm{C} 1$, a and b). The new corrected mean value for the remaining six laboratories were calculated for A (3.56 $\pm 0.99 \mu \mathrm{g} / \mathrm{mg}$; CV $27.9 \%)$ and B $(3.81 \pm 1.17 \mu \mathrm{g} / \mathrm{mg}$; CV $30.6 \%)$. Two laboratories determined the campesterol to cholesterol ratio within a $\pm 15 \%$ range of the corrected mean value.

In the second survey part thirteen participants were included with calculated results for sample A (3.71 $\pm 1.86 \mu \mathrm{g} / \mathrm{mg}$; CV 50.1\%) and B $(3.70 \pm 1.98 \mu \mathrm{g} / \mathrm{mg}$; CV $53.5 \%)$. One of the participants determined campesterol to cholesterol ratio within a $\pm 15 \%$ range of the mean value of all participants and five participants were identified as outliers (Fig. $2 \mathrm{C} 2$, a and e). The new corrected mean value for the remaining eight laboratories were calculated for A $(3.24 \pm 1.06 \mu \mathrm{g} / \mathrm{mg}$; CV $32.7 \%)$ and B (3.24 $\pm 1.01 \mu \mathrm{g} / \mathrm{mg}$; CV $31.3 \%)$. One of the remaining eight laboratories determined the campesterol to cholesterol ratio within a $\pm 15 \%$ range of the corrected mean value.

\subsubsection{Lathosterol to campesterol}

In the first survey part twelve participants were included with calculated results for sample A $(0.45 \pm 0.49 \mu \mathrm{g} / \mu \mathrm{g}$; CV 109.6\%) and B $(0.52 \pm 0.57 \mu \mathrm{g} / \mu \mathrm{g} ; \mathrm{CV} 109.2 \%)$. One of the participants determined lathosterol to campesterol ratio within a $\pm 15 \%$ range of the mean value of all participants and six participants were identified as outliers (Fig. $2 \mathrm{D} 1$, a to f). The new corrected mean value for the remaining six laboratories were calculated for A $(0.35 \pm 0.09 \mu \mathrm{g} / \mu \mathrm{g}$; CV $26.2 \%)$ and B $(0.33 \pm 0.08 \mu \mathrm{g} / \mu \mathrm{g} ; \mathrm{CV} 24.1 \%)$. One laboratory determined the lathosterol to campesterol ratio within a $\pm 15 \%$ range of the corrected mean value.

In the second survey part fifteen participants were included with calculated results for sample A $(0.55 \pm 0.28 \mu \mathrm{g} / \mu \mathrm{g}$; CV $51.5 \%)$ and B $(0.54 \pm 0.30 \mu \mathrm{g} / \mu \mathrm{g} ; \mathrm{CV} 56.4 \%)$. Seven of the participants determined lathosterol to campesterol ratio within $a \pm 15 \%$ range of the mean value of all participants and two participants were identified as outliers (Fig. $2 \mathrm{D} 1$, a and b). The new corrected mean value for the remaining eight laboratories were calculated for A $(0.50 \pm 0.10 \mu \mathrm{g} / \mu \mathrm{g}$; CV $20.1 \%)$ and B ( $0.50 \pm 0.09 \mu \mathrm{g} / \mu \mathrm{g}$; CV $18.7 \%)$. Seven of the remaining eleven laboratories determined the lathosterol to campesterol ratio within a $\pm 15 \%$ range of the corrected mean value.

The coefficient of variation for the average value for the ratio of $5 \alpha$ - cholestanol, lathosterol- and campesterol to cholesterol were calculated to be between $31.4 \%$ and $96.5 \%$, respectively. The coefficient of variation for the average value for the ratio of lathosterol to campesterol were calculated between $51.5 \%$ to $109.2 \%$. Here is to notice that the ratio of lathosterol to campesterol shows even higher variations probably because of the analytical challenges. It is noteworthy that this survey revealed a high variation for ratios of NCS to cholesterol which prevents definition of resilient threshold values.

\section{Common discussion}

Unlikely to the other NCS assays, a target value for cholesterol could be reported in the present survey. For NCS assays the reported values could only be compared to the mean of values obtained from all participants or to a corrected mean value after removal of defined outlier. Thus, the true NCS analyte concentration could not be referred with certainty in any of these assays. Absolute reference methods have been developed for some analytes of diagnostic importance but for most analytes there is no "golden method" and we are thus restricted to compare results between different laboratories $[9,10]$.

However, with the collected survey data on hand it is not possible to sufficiently evaluate the best chromatographic method for cholesterol and NCS determination in plasma or serum. It can be generally considered that the quality of the present type of cholesterol and NCS analysis is directly dependent upon the skill and experience of the analyst, the limitations of the analysis method, the sample quality and sampling itself, as well as on the quality of the calibration material and used internal standards. Within the survey six different methods were used whereby all measurements dependent upon a critical chromatographic step, either gas- or liquid chromatography. Due to higher chromatographic efficiency and the reduced influence of matrix effects on ionisation and signal suppression, one would expect less analytical problems with gas chromatography compared to liquid chromatography. As ideal standard for cholesterol and NCS analysis it can be expected that the use of the same deuterium or ${ }^{13} \mathrm{C}$ isotope labelled molecule will result in the most precise mass spectrometric analysis. In respect to that it is to mention that the internationally accepted reference method for cholesterol is based on isotope-dilution mass-spectrometry combined with GC $[8,9,11-13]$.

The use of common quantified calibration material slightly improved the variation of reported results even since individual laboratories and the number of participants is different in the two survey parts. Here, three points should be emphasized: First of all, commercially available standards do not always have the required purity of $99.0 \%$ needed. Quality and purity of standard materials used should always be controlled if possible. In addition to this, the weighing of small milligram amounts is often challenging and is prone to generate errors. To avoid these effects of impurities and weighing, we recommend to quantify the cholesterol and NCS calibration solutions by GC-FID ( $5 \alpha$-cholestanol) before use. Lastly, calibration solutions independent if self-prepared or commercially sourced have a limited bench stability. Using the same solution over years might alter internal analyte concentrations. Therefore, used stock solutions should be newly prepared or re-quantified after a certain usage time by the above described method and old stock solutions should be analysed alongside the new for a short overlapping period.

\section{Conclusions}

We found surprisingly high variations in cholesterol and NCS concentrations obtained from analytical assays based on chromatographic separation. The participating laboratories specialized in lipid analysis reported astoundingly strong differences in the concentrations of neutral sterols in both survey parts. However, the use of common calibration materials slightly improved the variations in the reported concentrations, whereby still high variations were obtained in the second 
interventional survey part. In glance to this situation the evaluation of normal plasma or serum reference concentrations of neutral sterols is very challenging and nearly impossible. Furthermore, this survey gives evidence for the urgent need for quality control programs with interchange of samples between different laboratories in order to harmonize the chromatographic analysis of cholesterol and NCS from serum or plasma samples. In the future we intent to provide following ring trials for the quantification of cholesterol, oxidized cholesterols and neutral sterols based on chromatographic separation techniques and mass spectrometric or flame ionization detection methods.

\section{Fundings}

We gratefully acknowledge funding for materials, statistics, and travel expenses to the Foundation for Pathobiochemistry and Molecular Diagnostics, German Society of Clinical Chemistry and Laboratory Medicine, Germany. The work in University of Valencia was financed by CICYT-FEDER (AGL2008-02591-C02-01) and MINECO-FEDER (AGL2012-39503-C02-01). The work at Universitario Miguel Servet, Zaragoza, Spain was funded by Fondo de Investigación Sanitaria (FIS; PI15/01983). Work in Swansea was supported by the UK Biotechnology and Biological Sciences Research Council (BBSRC, grant numbers BB/ I001735/1 and BB/L001942/1).

\section{Acknowledgements}

The basic initiatives for the performance of these surveys were started during and the results presented at the annual meetings of the European Network for Oxysterol Research (ENOR; https://www. oxysterols.net/). Most of the survey participants are members of the ENOR group.

\section{References}

[1] I. Björkhem, T. Miettinen, E. Reihner, S. Ewerth, B. Angelin, K. Einarsson, Correlation between serum levels of some cholesterol precursors and activity of HMG-CoA reductase in human liver, J. Lipid Res. 28 (10) (1987) 1137-1143.

[2] T.A. Miettinen, R.S. Tilvis, Y.A. Kesaniemi, Serum cholestanol and plant sterol levels in relation to cholesterol metabolism in middle-aged men, Metabolism 38 (2) (1989) 136-140.

[3] R.S. Tilvis, T.A. Miettinen, Serum plant sterols and their relation to cholesterol absorption, Am. J. Clin. Nutr. 43 (1) (1986) 92-97.

[4] T.A. Miettinen, R.S. Tilvis, Y.A. Kesaniemi, Serum plant sterols and cholesterol precursors reflect cholesterol absorption and synthesis in volunteers of a randomly selected male population, Am. J. Epidemiol. 131 (1) (1990) 20-31.

[5] D. Mackay, P.J. Jones, Evaluation of methods for the determination of cholesterol absorption and synthesis in humans, Atherosclerosis 218 (2) (2011) 253-262.

[6] D.S. Mackay, P.J. Jones, Plasma noncholesterol sterols: current uses, potential and need for standardization, Curr. Opin. Lipidol. 23 (3) (2012) 241-247.

[7] D.S. Mackay, P.J. Jones, S.B. Myrie, J. Plat, D. Lütjohann, Methodological considerations for the harmonization of non-cholesterol sterol bio-analysis, J. Chromatogr. B Anal. Technol. Biomed. Life Sci. 957 (2014) 116-122.

[8] Bundesärztekammer, Richtlinie der Bundesärztekammer zur Qualitätssicherung laboratoriumsmedizinischer Untersuchungen, Deutsches Ärzteblatt 38 (2014) A1583-A1618.

[9] H. Gylling, P. Simonen, L. Kaipiainen, I. Wester, Methodological aspects of phytosterol measurements in biological samples, Curr. Med. Chem. (2018).

[10] C.T. Srigley, S.L. Hansen, S.A. Smith, A. Abraham, E. Railey, X. Chen, S.H. Chooi, L.M. Clement, M. Dao, A.R.F. Kia, B. Mitchell, M. Mogla, J.A.R. Ortiz, K. Persons, E. von Kries, G. Ware, J. Wubben, R. Cantrill, Sterols and stanols in foods and dietary supplements containing added phytosterols: a collaborative study, J. Am. Oil Chem. Soc. 85 (2018) 247-257.

[11] S.H. Edwards, M.M. Kimberly, S.D. Pyatt, S.L. Stribling, K.D. Dobbin, G.L. Myers, Proposed serum cholesterol reference measurement procedure by gas chromatography-isotope dilution mass spectrometry, Clin. Chem. 57 (4) (2011) 614-622.

[12] I. Björkhem, R. Blomstrand, S. Eriksson, O. Falk, A. Kallner, L. Svensson, G. Ohman, Use of isotope dilution-mass spectrometry for accuracy control of different routine methods used in clinical chemistry, Scand. J. Clin. Lab. Invest. 40 (6) (1980) 529-534.

[13] I. Björkhem, R. Blomstrand, L. Svensson, Serum cholesterol determination by mass fragmentography, Clin. Chim. Acta 54 (2) (1974) 185-193. 